\title{
Evaluación estructural de paneles de soporte para viviendas usando mallas plásticas como refuerzo principal
}

Structural evaluation of support panels for housing using plastic meshes as main reinforcement.

\author{
${ }^{1}$ Ernesto Pérez Cerezález ${ }^{\mathrm{a}},{ }^{1}$ Awaleh Kaireh Youssouf Djibouti ${ }^{\mathrm{a}},{ }^{1}$ Luis Zulueta Martínez ${ }^{\text {a }}$
}

\section{RESUMEN}

Se presenta la evaluación estructural de paneles de ferrocemento con mallas fabricadas con polímeros para utilizarlos en viviendas económicas de una planta. El trabajo pretende la utilización de productos de producción local en Ciego de Ávila y Camagüey, tales como, las mallas plásticas y platinas metálicas, conformar paneles nervados y realizar ensayos a los materiales y a los elementos con ellos construidos. Primeramente se seleccionan los materiales cuyas características se ajustan a las propiedades propias del ferrocemento. Las mallas, las barras lisas y platinas se ensayan a tracción determinando su resistencia. Se construyen paneles nervados conformados con malla plástica y barras lisas y se ensaya a flexión obteniéndose las deformaciones y la carga para las que aparecen las primeras fisuras. Se modelan los paneles vertical y horizontalmente aplicando métodos digitales y se obtienen las fuerzas interiores producidas por el sistema de cargas impuesto. Se comparan los resultados de los modelos con los ensayos corroborándose la posibilidad de utilización de este sistema en paredes portantes y cubierta con determinadas ventajas para la construcción de viviendas, sobre los métodos tradicionales.

Palabras clave: mallas plásticas, paneles nervados, evaluación estructural, ferrocemento.

\begin{abstract}
The structural evaluation of ferrocement panels is presented with meshes manufactured with polymers to use them in economic housings of a plan. The work seeks the use of products of local production in Ciego of Ávila and Camagüey as the plastic meshes and metallic plates, to conform nervous panels and to carry out rehearsals to the materials and the elements with them built. Firstly the materials are selected whose characteristic they are adjusted to the properties characteristic of the ferrocement. The meshes, the flat bars and plates are rehearsed to traction determining their resistance. Nervous panels are conformed with plastic mesh and flat bars and it is rehearsed to flexion being obtained the deformations and the load for those that the first fissures appear. The vertical panels are modeled and horizontally applying digital methods and the interior forces are obtained taken place by the system of loads tax. The results of the models are compared with the rehearsals being corroborated the possibility of use of this system in walls ambles and covered with certain advantages for the construction of housings, on the traditional methods.
\end{abstract}

Keywords: plastic meshes, nervous panels, structural evaluation, ferrocement. 


\section{INTRODUCCIÓN}

El ferrocemento se ha utilizado en el mundo y en Cuba a partir de 1981, en la construcción de viviendas, como una opción válida, por su resistencia, por la rapidez con que se prefabrican los paneles portantes y la facilidad con que se manipulan. En esa utilización ha demostrado sacar ventajas relativas a las construcciones tradicionales y aún a las prefabricadas de hormigón armado. Las losas cajón que se han utilizado en Cuba tienen como acero fundamental estructural, un tipo de malla de acero galvanizado que generalmente se importa. Como acero de esqueleto se utilizan barras lisas y corrugadas de pequeño y mediano calibre. (Wainshtok, 2008)

Las producciones locales de malla plástica de aceptable resistencia, elasticidad y rigidez relativa, así como los subproductos en forma de platinas de las industrias locales de Ciego Ávila y Camagüey han motivado a varios investigadores a estudiar estos materiales para valorar su utilización efectiva en la construcción de viviendas.

Necesidad de utilizar producciones locales como materiales alternativos para la construcción de viviendas económicas.

Si se realizan ensayos de resistencia a mallas plásticas y platinas metálicas producidas en Ciego de Ávila y Camagüey se comparan con los valores que requieren estos materiales en modelos estructurales digitales para soportar determinadas cargas entonces podrían conformarse paneles con garantía estático resistente.

Evaluación estructural de paneles de ferrocemento con mallas plásticas de producción local para paneles portantes en viviendas.

\section{MATERIALES Y MÉTODOS}

En la investigación se utilizan los ensayos a tracción y flexión para comprobar la resistencia de los materiales a utilizar, sobre todo la malla plástica con que se sustituye la metálica que comúnmente usa el ferrocemento. Para desarrollar los ensayos se usa una máquina de ensayos universales Ibertest certificada de la Universidad de Camagüey, los ensayos a flexión en el panel fueron realizados con equipos de medir la resistencia y la defección de la ENIA de Ciego de Ávila. Se utilizó la modelación estructural como método fundamental para simular digitalmente la estructura y las acciones que enfrenta, se utilizan además métodos actualizados de diseño de ferrocemento, las normas vigentes de la ACI y se usa el Programa Staad Pro. 2008, para modelar y cualificar el análisis de la carga sísmica en la vivienda. Finalmente se comparan los estados tensionales que se logran en los ensayos con los del modelo para demostrar la factibilidad estructural de la malla y la platina como materiales sustitutos.

\section{RESULTADOS}

Para realizar la evaluación de la malla, se ha tenido en cuenta varios tipos fabricadas por el combinado CEPIL perteneciente a la provincia de Ciego de Ávila que fabrica mallas plásticas con desechos de materia prima y una malla que se ha utilizado en la cría de alevines en Santa Cruz del Sur pero puede resultar para todas las provincias del país una posibilidad de encontrar una alternativa viable que sustituya a los paneles de ferrocemento existentes con las mallas hexagonales, electrosoldadas y tejidas de importación por mallas plásticas. 


\section{Características de los materiales a utilizar.}

\section{Mallas construidas con polímeros (plásticas).}

Se analizaron 5 tipos de mallas, 4 tipos de barras lisas, 4 tipos de platina y un cable plástico cuyas características se detallan a continuación.

Las mallas escogidas en la Fábrica de cepillo y brocha para un primer análisis son las siguientes:

1. Malla tipo 1: Plástica tipo peerle azul, Color: azul, Material: Plástico duro. Tipo: Romboidal, Diámetro del alambre: $1.8 \mathrm{~mm}$, Abertura de malla: $25 \times 28 \mathrm{~mm}$.

2. Malla tipo 2: Plástica tipo peerle azul, Color: azul, Material: Plástico blando Tipo: Romboidal, Diámetro del alambre: $1.5 \mathrm{~mm}$, Abertura de malla: $35 \times 28 \mathrm{~mm}$.

3. Malla tipo 3: Plástica tipo peerle, Color: negro, Material: Plástico duro Tipo: Romboidal, Diámetro del alambre: $3 \mathrm{~mm}$, Abertura de malla: 48 x $48 \mathrm{~mm}$.

4. Malla tipo 4: Plástica hexagonal, Color: azul verdoso, Material: Plástico blando Tipo: hexágono irregular, Área del alambre: $2.2 \mathrm{~mm}$ X $1.9 \mathrm{~mm}$ (irregular), Abertura de malla: 13 $\mathrm{mm} \times 26 \mathrm{~mm}$.

5. Malla tipo 5 (figura 1) Tipo de malla: Plástica tipo peerle Color: negro Material: Plástico duro Tipo: Romboidal, Área del alambre: 2.5 mm X 1.5 mm Abertura de malla:26X28 mm.

Después de valorar los 5 tipos se desechan la 2, por ser un material muy blando, la 3 por tener una abertura de malla que está en el límite de las utilizadas en las mallas de ferrocemento usualmente, la 4 por ser de un material blando y se escogen la 1 y la 5 por cumplir a simple vista con las características técnicas que exige la malla de ferrocemento y ser de un material mucho menos deformable que el resto. Finalmente se someten a ensayos la malla tipo 1 y tipo 5. Por razones de espacio solo aparecerán los ensayos de la Tipo 5, por ser la menos deformable y mayor resistencia a tracción y por tanto la escogida para hacer los paneles.

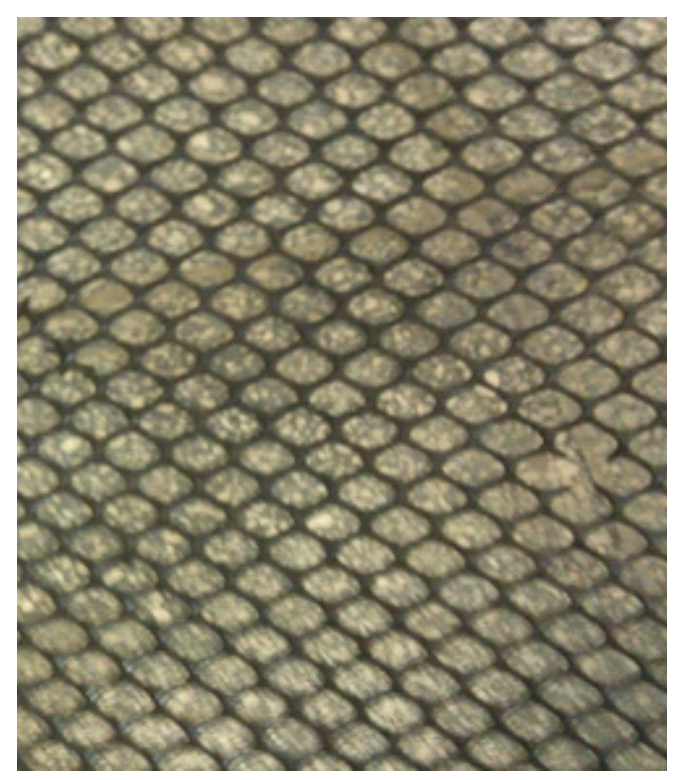

Figura 1. Malla plástica tipo 5

\section{Ensayos a los materiales. Ensayos a tracción a} las mallas.

Se realiza el ensayo a tracción de las 2 mallas en una máquina de ensayo universal Ibertest de los laboratorios de estructuras. Los resultados por tipo de malla se muestran a continuación, la fuerza a tracción se da en toneladas fuerza y corresponde al inicio de la fluencia. 
Tabla 1. Resultados de los ensayos a tracción a la malla tipo 1

\begin{tabular}{ccc}
\hline Ensayos & Fuerza a tracción & Posición \\
\hline 1 & 0.1 & Vertical \\
2 & 0.1 & Vertical \\
3 & 0.09 & Vertical \\
4 & 0.08 & Vertical \\
5 & 0.09 & Vertical \\
6 & 0.1 & Vertical \\
7 & 0.09 & Vertical \\
8 & 0.1 & Vertical \\
9 & 0.08 & Vertical \\
10 & 0.1 & Vertical \\
\hline
\end{tabular}

La carga promedio que reflejan los ensayos (tabla 1) donde comienza la fluencia es: 0.093 ton: 0.93 $\mathrm{kN}$.

La malla es romboidal por lo que la fuerza que resiste el cable hay que afectarla por el coseno del semiángulo que se produce entre los hilos verticales de la malla, en este caso el ángulo es 44.90 y el coseno será de 0.707 .

Entonces la resistencia de fluencia del cable es RA: FTF/0.707X6 XAa

Donde: RA : Resistencia al comenzar la fluencia de un cable de la malla.

6: Nro de cables que toma la mordaza de la máquina de ensayos (Figura 2.)

0.707: Coseno del ángulo que forma la el lado del rombo de la malla con la vertical Aa: Área de los alambres plástico de la malla Entonces RA: 32 MPa.(Malla Tipo 1)
Tabla 2. Resultados de ensayos de la malla tipo 5

\begin{tabular}{ccc}
\hline Ensayos & Fuerza a tracción & Posición \\
\hline 1 & 0.1 & Vertical \\
2 & 0.1 & Vertical \\
3 & 0.09 & Vertical \\
4 & 0.09 & Vertical \\
5 & 0.09 & Vertical \\
6 & 0.08 & Vertical \\
7 & 0.09 & Vertical \\
8 & 0.09 & Vertical \\
9 & 0.1 & Vertical \\
10 & 0.08 & Vertical \\
\cline { 2 - 3 } & &
\end{tabular}

La carga promedio que reflejan los ensayos (tabla 2) donde comienza la fluencia es: 0.091 ton: 0.91 $\mathrm{kN}$.

La malla es romboidal por lo que la fuerza que resiste el cable hay que afectarla por el coseno del semiángulo que se produce entre los hilos verticales de la malla, en este caso el ángulo es 44.90 y el coseno será de 0.707 .

Entonces la resistencia de fluencia del cable es RA: FTF/0.707X 6 XAa (A)(Wainstohk, 2009) La diferencia entre la malla 5 y la 1 está en el grosor del alambre que en el caso anterior era de $7.065 \mathrm{~mm} 2$ y en el tipo 5 es de $3.75 \mathrm{~mm} 2$. Los elementos que conforman la fórmula $\mathrm{A}$ son similares a lo expresado en los cálculos del tipo 1 solamente cambia la fuerza de fluencia y el área del alambre aplicando la fórmula (A) y sustituyendo RA: 57.2 MPa. 
Este valor es más de la mitad de la resistencia de algunas mallas metálicas que recoge la bibliografía.

El ensayo con la malla colocada horizontalmente da resultados similares en ambos casos.(Macías y Artola, 2010)

Las probetas utilizadas para la malla son de 30 $\mathrm{cm}$ de largo por $10 \mathrm{~cm}$ de ancho, según la mordaza utilizada, la más ancha con muescas de agarre adecuadas, con $12 \mathrm{~cm}$ de probeta longitudinal libre y el resto de agarre. (Figura 2). Como se nota quedan 6 alambres en el ancho.(Bedoya y Alvarez, 2009)

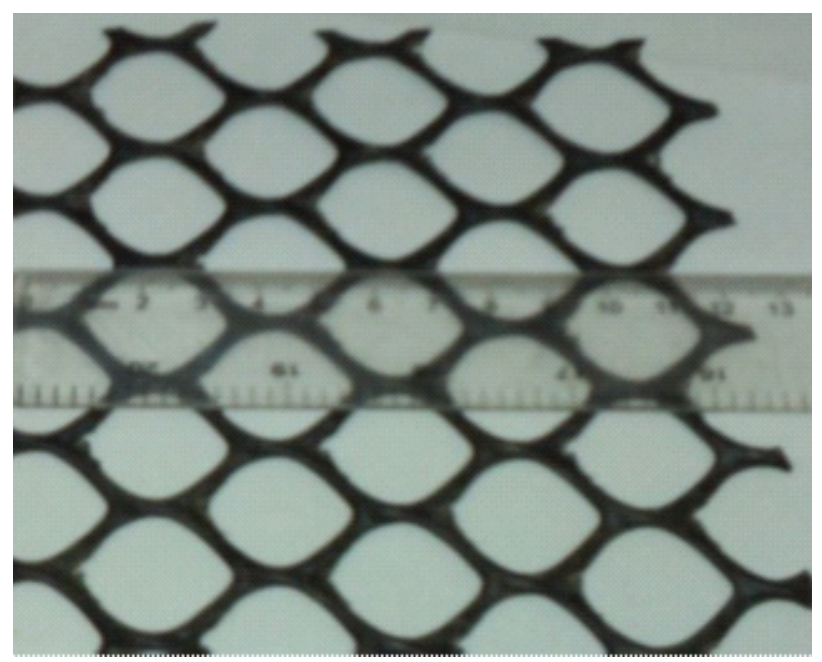

Figura 2. Probeta de malla, detalle de medición de ancho.

\section{Platinas.}

Se realizan ensayos a las platinas de $2 \mathrm{~mm} \mathrm{X}$ $11 \mathrm{~mm}$ (Tabla 2.4), $1.5 \mathrm{~mm}$ X 12,5 mm y $2 \mathrm{~mm} \mathrm{X}$ $18 \mathrm{~mm}$, las probetas usadas son de $300 \mathrm{~mm}$.

Tabla 3. Resultados de los ensayos a la platina de 2 X $11 \mathrm{~mm}$.

\begin{tabular}{ccc}
\hline Ensayos & $(\mathrm{T})$ & $\begin{array}{c}\text { Fuerza a } \\
\text { tracción }(\mathrm{KN})\end{array}$ \\
\hline 1 & 0.50 & 5.0 \\
2 & 0.52 & 5.2 \\
3 & 0.50 & 5.0 \\
4 & 0.50 & 5.0 \\
5 & 0.60 & 6.0 \\
6 & 0.60 & 6.0 \\
7 & 0.60 & 6.0 \\
8 & 0.53 & 5.3 \\
\hline
\end{tabular}

El promedio que alcanza la fuerza a tracción es de $5.46 \mathrm{kN}$, el área de la platina es de $22 \mathrm{~mm} 2$ y por tanto la Resistencia a tracción obtenida RA: 248 $\mathrm{MPa}$, en las platinas de $1.5 \mathrm{X} 12.5 \mathrm{~mm}$ se obtiene una RA : $277 \mathrm{MPa}$ y en la de 2 X $18 \mathrm{~mm}$ la RA : $256 \mathrm{MPa}$ que son resultados esperados en este tipo de plancha en todos los casos. Como lo novedoso del trabajo son las mallas construidas con polímeros y los resultados con las planchas son los que da el fabricante con menos de un $5 \%$ de desviación se trabaja en el panel con la de 2 x 18 de RA: $256 \mathrm{MPa}$ que es similar a la resistencia que alcanza el alambrón de $6 \mathrm{~mm}$ que es uno de los que se utiliza en el ferrocemento.(Dorta, 2011)

Ensayo a un panel con malla plástica para pared portante y cubierta propuesto para una vivienda.

Características del panel.

El panel propuesto tiene un largo de $2.5 \mathrm{~m}$ con $0,40 \mathrm{~m}$ de ancho con 3 nervios espaciados a $0.175 \mathrm{~m}$ y de $0.075 \mathrm{~m}$ de altura tanto los nervios como el panel tienen un espesor de $3 \mathrm{~cm}$, como se muestra en la Figura 4.(Díaz y Pérez, 2013) 


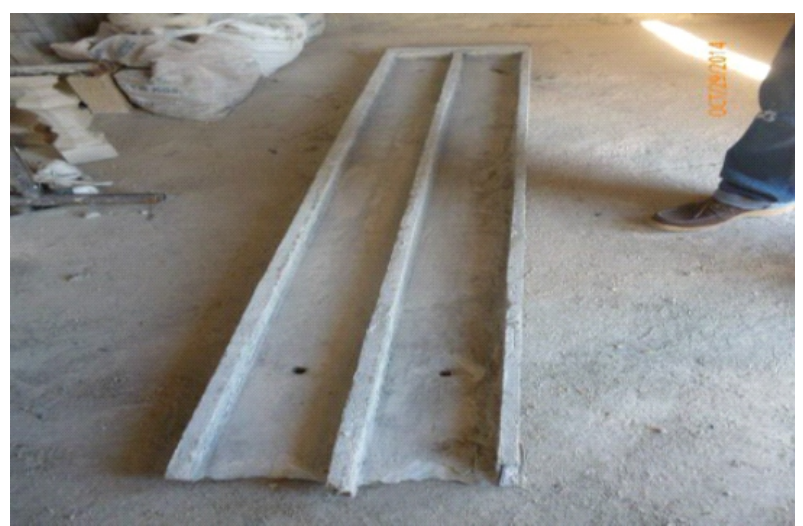

Figura 4. Panel construido con malla plástica tipo 5.

El panel fue construido por una cooperativa en Ciego de Àvila llamada "Los Jiménez"con la intención de ensayarlo como una primera aproximación para sustituir los bloques de paramento vertical y para usarlo como cubierta o entrepiso. El MSc. Julio Fernández, de dicha cooperativa, contactó con el CECODEC (Centro de estudios de la Universidad de Camagúey) para realizar la investigación de manera conjunta.

El panel fue construido utilizando 2 mallas plásticas tipo 5 (ONN, 2007) y barras lisas de 6 $\mathrm{mm}$, cinco barras longitudinales, una en el centro, cuatro encima y debajo en los nervios y barras transversales en los extremos(estas barras tienen resistencia similares a las platinas propuestas y ensayadas). En la figura 5 se ven detalles de la malla y las barras durante la construcción del panel.

Ensayo a flexión del panel.

El panel fue ensayado en la ENIA de Ciego de Àvila con adición de carga por tiempo de $50 \mathrm{Kgf}$ como se muestra en la Figura 5 apoyado en los extremos con una luz libre de $2.50 \mathrm{~m}$. Al panel se le instalaron deflectómetros (figura 5) para valorar su deformación y observar durante 2 horas la aparición de grietas visibles (mayores de $0.5 \mathrm{~mm})$.

En la tabla 6 se muestran los resultados de los ensayos por tiempo y las mediciones detectadas en los 2 deflectómetros.
La capacidad de los deflectómetros impidió sobrepasar los $11 \mathrm{~mm}$ de flexión en el centro cuando llegaba la carga a $490 \mathrm{Kgf}(4.9 \mathrm{kN})$ y todavía sin llegar a la fluencia.

Las grietas que aparecen en el panel tanto transversales como longitudinales, son apenas visibles (cercanas a $0.5 \mathrm{~mm}$ ). Uno de los autores de este trabajo observó el panel 48 horas después de retirada la carga máxima y no apreció grietas de ningún tipo lo que quiere decir que puede considerarse para ese panel que para cargas menores de $500 \mathrm{Kgf}(5 \mathrm{kN})$ está en régimen elástico. Como el panel tiene un área efectiva de $1 \mathrm{~m} 2$ la carga que resistió es de $5 \mathrm{kN} / \mathrm{m} 2$ sin llegar a completar el 2 do estado o estado elastoplástico.

La carga que soporta un panel de estas características en cubierta teniendo en cuenta 1.2 $\mathrm{CP}+1.6 \mathrm{CU}$ con caída libre es de aproximadamente de $4 \mathrm{kN} / \mathrm{m} 2$ por lo que resistiría fácilmente esa carga.

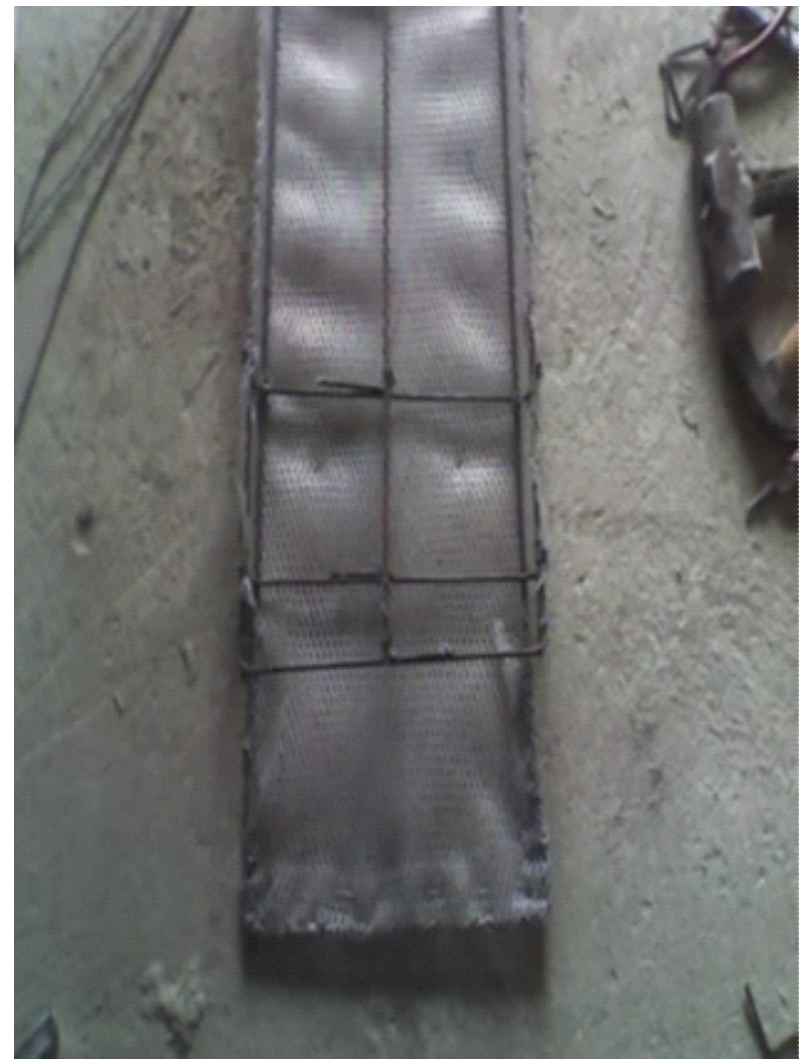

Figura 5. Detalles del panel antes de vertirse el mortero 


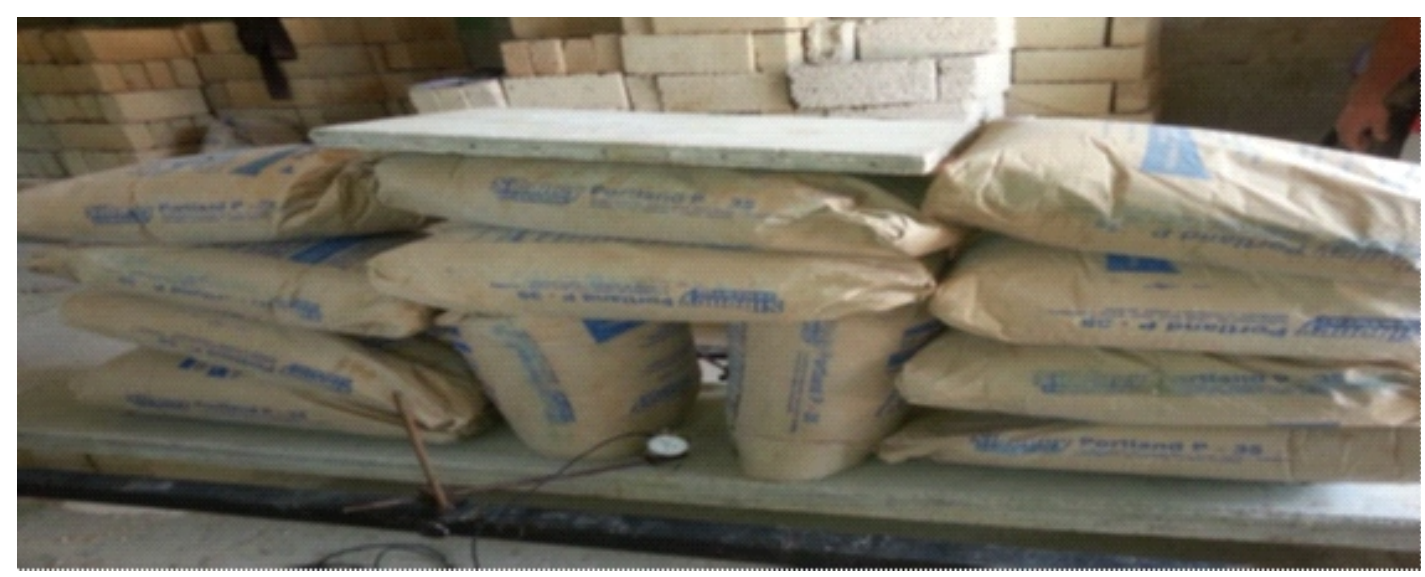

Figura 6. Panel cargado con $540 \mathrm{Kgf}(5.4 \mathrm{kN})$ sin entrar en fluencia con 4 deflectómetros colocados.

Tabla 6. Resumen de los resultados de las mediciones del ensayo a flexión del panel

\begin{tabular}{llll}
\hline $\begin{array}{l}\text { Secuencia de } \\
\text { lecturas }\end{array}$ & $\begin{array}{l}\text { Carga sobre la } \\
\text { placa(kg) }\end{array}$ & $\begin{array}{l}\text { Lectura } \\
\text { carátula } \\
\text { derecha(mm) }\end{array}$ & $\begin{array}{l}\text { Lectura carátula } \\
\text { 1zquierda(mm) }\end{array}$ \\
\hline 9.56 & 126 & 2.39 & 3.25 \\
10.15 & 249 & 5.48 & 3.28 \\
11.20 & 369 & 7.56 & 6.83 \\
12.00 & 490 & 9.87 & 10.00 \\
12.30 & 505 & 10.00 & 10.25 \\
\hline
\end{tabular}

La diferencia anuncia que es factible su utilización en cubierta y como pared portante donde la flexión es mucho menor.

Un panel de $2.50 \mathrm{~m}$ de altura es ideal como pared portante pero un tanto pequeño para cubierta de una vivienda por lo que se utilizarán paneles más grandes para modelar los paneles horizontales utilizando STAAD Pro con $4 \mathrm{~m}$ de luz en trabajo a flexión horizontal semejando la cubierta.

También se realizará un modelo vertical para comprobar que el panel resiste verticalmente la carga de la cubierta.

\section{Modelo estructural del panel.}

a. Modelo horizontal: El modelo horizontal se concibe utilizando como base un panel de $4 \mathrm{~m}$ de luz y $0.40 \mathrm{~m}$ de ancho con 3 nervios de $0.075 \mathrm{~m}$ de altura de nervio y un espesor de $0.03 \mathrm{~m}$ para todo el panel. La combinación de carga para la cubierta es como se muestra: Carga permanente G: $2.50 \mathrm{kN} / \mathrm{m}^{2}$. Carga de uso Q: $0.8 \mathrm{kN} / \mathrm{m}^{2}$ $1.2 \mathrm{G}+1.6 \mathrm{Q}: 4.28 \mathrm{kN} / \mathrm{m}^{2}$ 
Con esas cargas, las dimensiones del panel y las características del ferrocemento se entra al software profesional Staad Pro

Se obtiene el modelo geométrico siguiente así como las reacciones (figura 7)

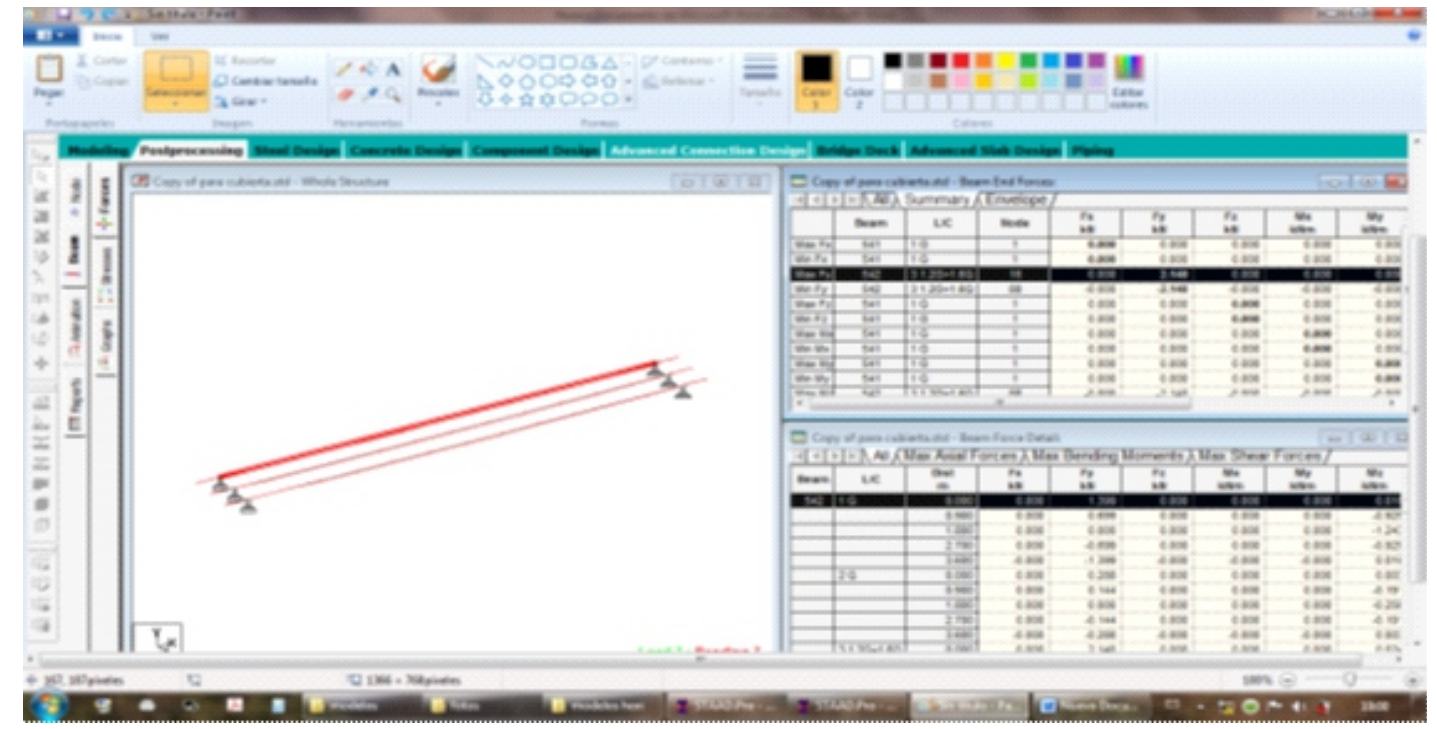

Figura7. Modelo estructural en la tabla del modelo aparecen las fuerzas en todos los ejes y los momentos.

Los momentos máximos en el centro son de alrededor de $3.8 \mathrm{kN}$-m y las tensiones a tracción máximas que genera el estado de carga son de $2.85 \mathrm{MPa}$, las compresiones son menores de $6 \mathrm{MPa}$.

b. Modelo vertical: Para el panel vertical se utilizan las mismas dimensiones que el panel ensayado en Ciego de Ávila, es decir $2.50 \mathrm{~m}$ x $0.40 \mathrm{~m}$ para un estado de carga similar que incluye la viga cerramento. Los modelos estructurales del Staad Pro se aprecian en la figura 8. De la figura 8 se infiere que el panel para la carga que lo requiere está a compresión, como se refleja en el estado tensional por colores, no existen tracciones (son positivas en la convención del software) y las compresiones que aparecen son muy pequeñas, fácilmente asimilables por el panel que aún con malla plástica puede resistir compresiones mayores de $8 \mathrm{MPa}$ fácilmente.

Se deduce que el momento por pandeo es mínimo (figura 8)

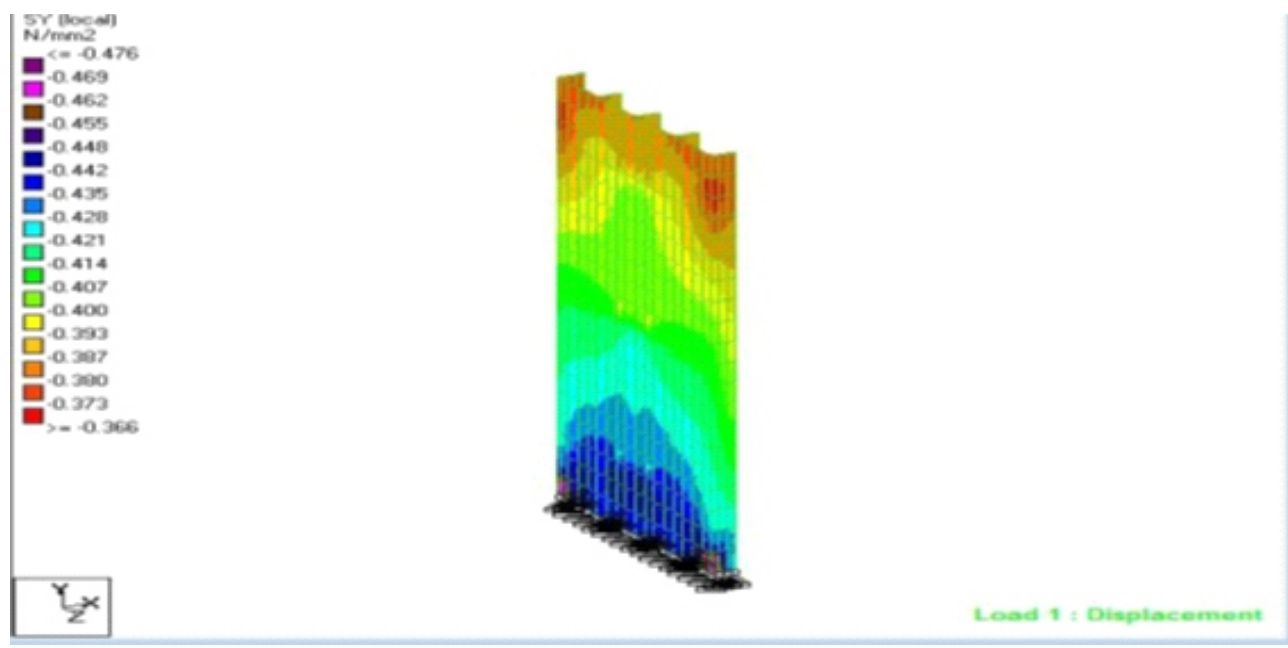

Figura 8. Estado tensional en el eje y 


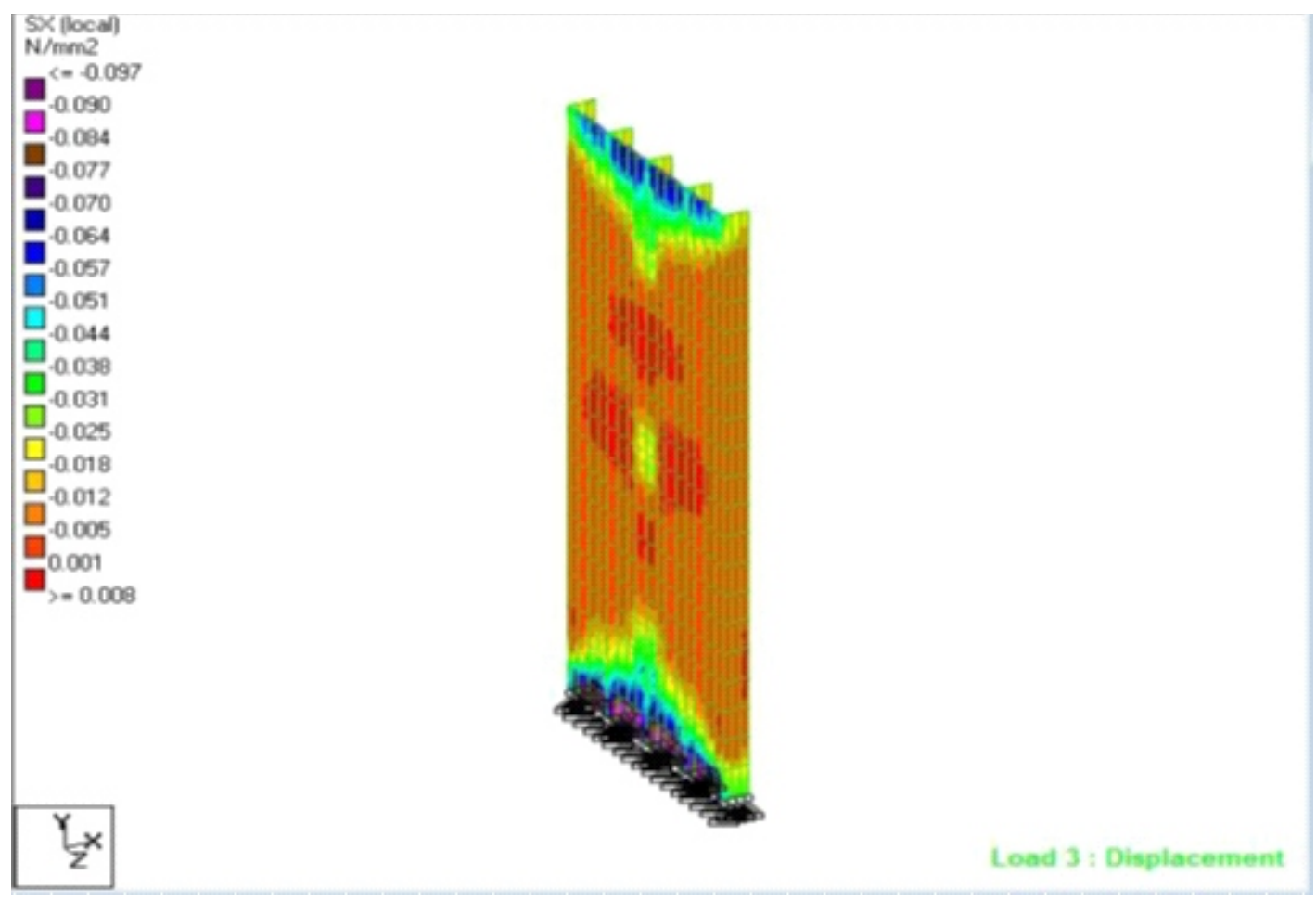

Figura 9. Estado tensional en el eje $x$

Como se aprecia solo aparecen tracciones en el centro y son de $0.001 \mathrm{MPa}$, lo que indica que el momento por pandeo es despreciable lo que corrobora que el panel ensayado en este trabajo con malla plástica y barras lisas es fácilmente utilizable como pared portante de una vivienda ya que no aparecen tracciones de consideración ante el estado de carga. Los ensayos a tracción a la malla indican que el panel de ferrocemento construido con ella y acero de esqueleto puede sobrepasar tracciones de $3 \mathrm{MPa}$ y por tanto no debe tener problemas para utilizarse como cubierta con $4 \mathrm{~m}$ de luz. (Wainstohk, 2008)

Sin embargo, aunque el único ensayo a flexión realizado dio una resistencia y rigidez del panel que indican su factibilidad portante horizontal y vertical es recomendable realizar más ensayos que lo demuestren fehacientemente. (Llanes, 2005)

Chequeo del momento resistente del panel con malla plástica

Conociendo el valor de la resistencia a tracción de la malla plástica y de las barras lisas o platinas, puede aplicarse un método práctico de diseño de ferrocemento para determinar con bastante aproximación el Momento Resistente de cualquier sección de ferrocemento. Si se conoce la posición de la línea neutra en una sección y la posición de la malla y el acero a tracción puede determinarse el momento resistente que aporta la malla y el acero en la zona traccionada. (Wainstohk, 2008)

Se consideran 2 mallas a tracción por cada uno de los 3 nervios de la losa que según la abertura de la malla incluye 3 alambres por nervio. La malla tipo 5 tiene una resistencia a tracción de los ensayos de 57.2 MPa con un área de los alambres de $3.75 \mathrm{~mm} 2$.

Utilizando una barra lisa de $6 \mathrm{~mm}$ en cada uno de los extremos inferiores de $320 \mathrm{MPa}$ de resistencia según los ensayos con un área de $28 \mathrm{~mm} 2$.

Simplificando el método de Olveras el MR: Momento de la malla + Momento del acero a tracción. 
M Malla: 3 alambres x $57.2 \mathrm{MPa}$ x $3.75 \mathrm{~mm} 2$ x $0.043 \mathrm{~m} \times 6$ mallas +3 barras lisas de $6 \mathrm{~mm} \times 320$ MPa x $28 \mathrm{~mm} 2$ × $0.085 \mathrm{~m}: 0.19 \mathrm{kN}-\mathrm{m}+2.28$ : $2.47 \mathrm{kN} . \mathrm{m}$.

Colocando 2 barras lisas en los nervios de los extremos se logra aumentar el momento resistente total en $1.52 \mathrm{kN} . \mathrm{m}$ lo que logra un momento total resistente de $4 \mathrm{kN} . \mathrm{m} \geq 3.8 \mathrm{kN}$.m actuante en el modelo horizontal.

$\mathrm{Si}$ en vez de las barras lisas se utilizan platinas de 2 x 18 mm cuyas resistencias a la tracción según los ensayos realizados en este trabajo son similares a los de las barras lisas se logran momentos resistentes un poco mayores (4.12 kN.m).

\section{CONCLUSIONES}

Se realizan ensayos a tracción a 2 tipos de malla escogidos, 3 tipos de platinas y 3 tipos de barras lisas obteniéndose la resistencia a tracción de cada uno de ellos. Es significativo que se obtienen resistencias a tracción de más de 50 MPa para las mallas ensayadas. Los aceros ensayados arrojan resistencias normales para ese tipo de material

Se realiza un ensayo a flexión a un panel nervado de ferrocemento construido con malla plástica tipo 5 y barras lisas de $6 \mathrm{~mm}$ obteniéndose cargas de prefluencia mayores de $5 \mathrm{kN}$ y pequeñas deformaciones lo que les da muchas posibilidades como paneles portantes verticales y horizontales. Aunque la ejecución de un solo ensayo limita la veracidad del resultado.

Se modelaron estructuralmente los paneles horizontales y verticalmente obteniéndose valores de las fuerzas interiores que al compararlas con los resultados de los ensayos indican la posibilidad de su uso como elementos portantes en viviendas.

\section{REFERENCIAS BIBLIOGRÁFICAS}

Bedoya, D, y Álvarez, D. ( 20009 ). Comportamiento de viviendas de ferrocemento para cargas cíclicas. Ingenierías de Medellín, Medellín, Colombia.8(15), 13.

Díaz, X. y Pérez, E. (2013). Elementos portantes de ferrocemento para edificio de vivienda antisísmica. Tesis de Maestría en Estructuras. Universidad de Camagüey, Camagüey, Cuba.

Dorta, R. (2011). Elementos nervados de ferrocemento [versión electrónica]. Recuperado el 23 de marzo de 2014, de h t t p : / / w w w . b e t s i m e . disaic.cu/secciones/ger_ma_03.htm.

Llanes, C. (2005). El ferrocemento una opción frente a los desastres [versión electrónica]. En IV Conferencia latinoamericana de ferrocemento. Recuperado el 24 de marzo de 2012, de http//www. cecat.com/ferrocemento.

Macías, J. y Artola , M (2010). Uso de las fibras orgánicas en paneles ligeros. En 10mo Simposio Internacional de Ferrocemento (FERRO 10) (pp. 3-8). La Habana, Cuba: [s.n.].

Oficina Nacional de Normalización (2007). Código de buenas prácticas para el ferrocemento con telas de malla de alambre y armaduras de esqueleto. Ciudad de La Habana, Cuba: Oficina Nacional de Normalización.

-Wainshtok, H. (2007). El ferrocemento en Cuba. En 5ta Conferencia latinoamericana y del caribe de Ferrocemento. (pp. 3-6). Quito, Ecuador: [s.n.]. 
Wainshtok, H. (2008). Ferrocemento. Diseño y Construcción (3ra ed.), Quito, Ecuador: Offset Abad Cía.

\section{CORRESPONDENCIA}

Mag. Ernesto Pérez Cerezalez

ernesto.cerezalez@reduc.edu.cu 